\title{
A NOVEL FUZZY NON-RADIAL DATA ENVELOPMENT ANALYSIS: AN APPLICATION IN TRANSPORTATION
}

\author{
Deepak Mahla*, Shivi Agarwal and Trilok Mathur
}

\begin{abstract}
The slack-based measure (SBM) DEA model is a non-radial model used to calculate the relative efficiency, input, and output targets of the different decision-making units (DMUs) based on their best peers or efficient frontier. The conventional SBM DEA model used crisp inputs and outputs. But, it can be observed in real-life problems that sometimes the available data is in linguistic forms such as "few", "many", "small", or missing data. The DEA technique is frontier based, and therefore, imprecise data may lead to untenable results. Fuzzy theory, which is already established to handle uncertain data, can overcome this problem. Furthermore, the sensitivity and stability analysis have been checked the robustness of fuzzy DEA models. In this study, sensitivity and stability analysis of the fuzzy SBM DEA has been performed. The lower and upper sensitive bounds for inputs and outputs variables have been obtained for both the inefficient and efficient DMUs to calculate the input and output targets. Finally, a real-life transportation problem for the validity of the study is presented for its depiction.
\end{abstract}

Mathematics Subject Classification. 03B52, 90C08, 90C31, 90C70, $03 \mathrm{E} 72$.

Received March 13, 2021. Accepted June 25, 2021.

\section{INTRODUCTION}

Charnes et al. [10] wrote a revolutionary paper on data envelopment analysis (DEA) [12] which is also one of the most cited articles of European Journal of Operational Research. It is a methodology to calculate the relative efficiency and computes the input and output targets, using multiple attributes of various decision-making units (DMUs). The computation of input and output targets makes DEA differ from other multi-criteria techniques. These targets are beneficial from the managerial point of view to improve the firms' relative efficiency. In recent years, there has been an exponential growth in the number of publications related to theory and applications of (DEA) [15]. Many researchers have integrated DEA with fuzzy theory to overcome its ineffectiveness on uncertain data. The uncertain data can incorporate errors in efficiency evolution. Therefore, the sensitivity analysis is used to check the robustness of the DEA models. Sensitivity and stability analysis often investigate the robustness of results to changes in the sample size, the number of variables in the analysis. The change in results with the variation of input data is known as sensitivity analysis, apparently done from the earliest days for different models. In DEA, Charnes et al. [11] firstly did the sensitivity analysis by varying the single input or

Keywords. Data Envelopment Analysis, fuzzy slacks-based measure model, credibility measure, decision-making units, sensitivity analysis.

Birla Institute of Technology and Science, Pilani, India.

*Corresponding author: p20170024@pilani.bits-pilani.ac.in, deepakmahlabits@gmail.com

(C) The authors. Published by EDP Sciences, ROADEF, SMAI 2021 
output. After that, many researchers like Charnes and Neralic [9], Neralic and Wendell [34,35] proposed several ways for sensitivity analysis of DEA models. Real-life situations are very different, and the available data in real life can be complex, qualitative, and incomplete. Conventional DEA models cannot handle these situations, and some integrated DEA models are required to handle these types of data.

To handle these types of data, many researchers like Sengupta [40], Banker [7], and Olesen and Petersen [36] employed the probability theory to establish stochastic DEA models. Sometimes, when the available data is in qualitative form, it is transformed into numerical data by inviting subject or domain experts to evaluate the degree of confidence in all possible situations. Subsequent evidence from various studies in different settings around the world shows that people routinely inflate small probabilities when answering these types of questions [21]. Probability theory requires large samples because the smaller the experts' views, the larger the ideas' variance. Sometimes, experts' sample size is small due to economic reasons, or not all experts respond. Therefore, in this case, probability theory is not the best technique to handle qualitative data. The fuzzy set theory (FST) [47] handled this situation more effectively and it has been expanded to deal with the concept of partial fact ranging from correct to incorrect. FST has become the fundamental tool for handling imprecision or vagueness, aiming at tractability, robustness, and low-cost solutions for real-world problems. Over the three-decade of research, there are mainly six primary categories [14] of fuzzy DEA models, namely, the tolerance approach, the $\alpha$-level based approach, the fuzzy ranking approach, the possibility approach, the fuzzy arithmetic, and the fuzzy random/type-2 fuzzy set [23]. The $\alpha$-level-based approach is the most commonly used technique to solve the fuzzy DEA model. But it calculates the relative efficiency in the interval form (i.e., lower bound efficiency and upper bound efficiency), and therefore ranking of the DMUs becomes another task. In this approach, input and output are obtained in the form of fuzzy numbers and intervals. The DEA model will be useful only when managers easily understand the results obtained from these models. But, obtained results from the $\alpha$-level-based approach are complex and need to be simplified. The possibility approach is also a widely used approach to solve fuzzy DEA models. The relative efficiency obtained from the possibility approach has a single value at a given $\alpha$ value. However, it has an inadequacy of self-duality, which is necessary for both theoretically and practically. Liu [26] introduced the credibility measure, which is self-dual in nature, works convincingly by managing human degree numerically. In this study, the crediblistic approach on the non-radial SBM DEA [42] model is taken into account. Wen et al. [44] also used the credibility measure to solve the CCR DEA model. The model is apt for the constant return to scale, but both primal and dual forms of the CCR model are required to measure the relative efficiency and efficient targets. Lovell [31] analyzed that DEA should be a variable return to scale to become translational invariable. Thus, the CCR DEA model is not translational invariable and should not be used when negative data exist. Therefore, the SBM DEA model has been selected to compute the relative efficiency, input, and output targets for this study. Other properties of the SBM DEA model can be studied in Article [42]. The SBM DEA model computes relative efficiency and slacks in inputs/outputs simultaneously. The slacks are necessary to compute input and output targets. Due to the above properties, the SBM model has been very applicable in various applications. Many researchers integrate fuzzy set theory with the SBM DEA model to handle the uncertain data and explore this model in many applications. Jahanshahloo et al. [19] did an initial study in which they solved the fuzzy SBM DEA model using a membership function to determine the relationship among two triangular fuzzy numbers. Saati et al. [37] applied the $\alpha$-cut approach to convert the fuzzy linear programming problem to an interval programming problem on the fuzzy SBM DEA model. Recently, Agarwal et al. [3] applied the possibility approach to solving the fuzzy SBM DEA model. Wanke et al. [43] used fuzzy DEA and stochastic DEA to analyze the Angolan banks and concluded that efficiency scores are similar to some extent when compared within the ambit of stochastic DEA and fuzzy DEA models. Zhou et al. [49] constructed a new evaluation fuzzy DEA model for portfolio management. Arana et al. [5] proposed an exciting approach, which focused not just on the computation of efficiency scores but also on the input and output improvements. These improvements help managers with some helpful information on the variables, and thus they can concentrate their efforts by which the progress can be possible. Arana et al. [6] proposed a radial twophase input-oriented FDEA approach on the trapezoidal fuzzy numbers. The approach explicitly implements the LU-fuzzy partial order. They also converted the FFLP model into a multi objective optimization problem using 
TABLE 1. Comparison with existing approaches.

\begin{tabular}{|c|c|c|c|c|c|c|c|}
\hline Properties & Lotfi et al. [30] & Sanei et al. [38] & Wen et al. [45] & Wen et al. [46] & Nerali et al. [35] & Arana et al. [5] & Proposed approach \\
\hline Efficiency evaluation & BCC-DEA & BCC-DEA & BCC-DEA & BCC-DEA & BCC-DEA & SBM-DEA & SBM-DEA \\
\hline Environment & Fuzzy & Fuzzy & Fuzzy & Fuzzy & Crisp & Fuzzy & Fuzzy \\
\hline $\begin{array}{l}\text { Unit Invariance } \\
\text { Model }\end{array}$ & $x$ & $x$ & $x$ & $x$ & $x$ & $\checkmark$ & $\checkmark$ \\
\hline Translational Model & $x$ & $x$ & $x$ & $x$ & $x$ & 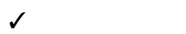 & $\checkmark$ \\
\hline $\begin{array}{l}\text { Credibilistic } \\
\text { framework }\end{array}$ & $x$ & $x$ & $\checkmark$ & $x$ & $x$ & $x$ & $\checkmark$ \\
\hline $\begin{array}{l}\text { Sensitivity \& stability } \\
\text { analysis }\end{array}$ & $\checkmark$ & $\checkmark$ & $\checkmark$ & $\checkmark$ & $\checkmark$ & $x$ & $\checkmark$ \\
\hline $\begin{array}{l}\text { Simultaneous } \\
\text { computation of } \\
\text { efficiency \& efficient } \\
\text { goals }\end{array}$ & $x$ & $x$ & $x$ & $x$ & $x$ & $x$ & $\checkmark$ \\
\hline
\end{tabular}

the fuzzy Pareto solutions and solved the problem utilizing the lexicographic weighted Tchebycheff method. Also, for each DMU, a new fuzzy efficiency measure and a fuzzy target has been measured. Heydari et al. [18] proposed a fully fuzzy approach to handle uncertainty in the data and solved this model using a lexicographic approach that gives efficiency scores in interval numbers. And then, this obtained multi objective model is used to calculate compute the 14 Iranian airlines efficiencies. Pankaj et al. [17] proposed portfolio efficiency evaluation using BCC-DEA and RDM model under fuzzy environments. The application of the model is shown in using superior risk measures of value at risk and conditional value at risk under a credibility measure. However, so far, no study exists that can simultaneously tackle uncertain data, efficiency, and efficient goals. The present study focuses on the fuzzy SBM DEA model's sensitivity and stability analysis, which has been solved after transforming into crisp linear programming models using credibility measures. The two theorems for inefficient DMUs and one theorem for efficient DMUs will be proposed in Section 3. These theorems will be used to calculate the input and output targets for the DMUs. Further, the proposed study's application has been shown for the transport data of State Transport Undertakings (STUs). The study's novelty is to compute relative efficiency, input, and output targets using a single model for missing data. The rest of the paper is organized as follows; Section 2 recalls the basic SBM, fuzzy SBM DEA model, and the credibility measure to solve the fuzzy SBM DEA model. Section 3 describes the sensitivity and stability analysis for the fuzzy SBM DEA model. A numerical example has been illustrated for the comparison in Section 4. In Section 5, an application is given based on the proposed methodology in which the relative efficiency as well as the lower and upper bounds of the inefficient and efficient State Transport Undertakings (STUs) for which they either become efficient or remain efficient. In the end, the conclusion about the proposed methodology is given (Tab. 1).

\section{Conceptual Framework}

This section discusses the SBM DEA model, fuzzy numbers, fuzzy SBM DEA model, credibility measure, some basic results and definitions which will be play the important part in the study.

\subsection{SBM DEA model}

Tone [42] proposed a non-radial DEA model, named as SBM DEA model, in 2001. The SBM DEA model deals directly with the input and output slacks and has some significant practical properties. The SBM DEA model's objective function is such that it gives relative efficiency and the input and output slacks simultaneously. This model is units invariant in nature and monotone decreasing with respect to input excess and output shortfall. Consider there are $m$-inputs, $n$-outputs, $r$-number of DMUs, $x_{i z}=$ amount of $i$ th input used by $z$ th DMU, $y_{j z}=$ amount of $j$ th output used by $z$ th DMU, $s_{i z}^{-}=$slack in the $i$ th input of the $z$ th DMU, $s_{j z}^{+}=$slack in the $j$ th output of the $z$ th DMU, and $\lambda_{o z}$ are intensity variables. Then, the SBM DEA model with the variable 
return to scale for $\mathrm{DMU}_{z}$ is given by,

$$
\begin{array}{cl}
\min \quad \rho_{z}= & \frac{1-\frac{1}{m} \sum_{i=1}^{m} s_{i z}^{-} / x_{i z}}{1+\frac{1}{n} \sum_{j=1}^{n} s_{j z}^{+} / y_{j z}} \\
\text { subject to: } \quad & \sum_{o=1}^{r} \lambda_{o z} x_{i o}+s_{i z}^{-}=t x_{i z} \quad \forall i=1, \cdots, m \\
& \sum_{o=1}^{r} \lambda_{o z} y_{j o}-s_{j z}^{+}=t y_{j z} \quad \forall j=1, \cdots, n \\
& \sum_{o=1}^{r} \lambda_{o}=1 \\
& \lambda_{o z} \geq 0, s_{i z}^{-} \geq 0, s_{j z}^{+} \geq 0, \quad \forall o=1, \cdots, r .
\end{array}
$$

Sometimes, the data available in real life is in qualitative form, or the data is missing. The traditional SBM DEA model does not work on missing data. This problem is handled by converting missing data into fuzzy numbers using the algorithm given in Section 4. The fuzzy numbers, credibility measure, and fuzzy SBM DEA models will be discussed in the next subsection.

\subsection{Fuzzy number}

The fuzzy set and fuzzy number were introduced by Zadeh [47] to handle the vague data in a precise way. A fuzzy set on universal set $M$ is defined by $\tilde{M}=\left\{\left(x, \mu_{\tilde{M}}(x)\right) \mid x \in M ; \mu_{\tilde{M}}(x) \in[0,1]\right\}$ in which $\mu_{\tilde{M}}(x)$ is called the membership function of the fuzzy set. Fuzzy numbers are the generalization of real numbers whose weight function lies between 0 and 1 , and this weight function is known as the membership function. The fuzzy numbers are a special kind of fuzzy sets defined on real numbers $R$. The fuzzy numbers satisfy the following properties:

(1) Fuzzy numbers are normal (i.e., $\exists x \in R: \mu_{\tilde{M}}(x)=1$ ).

(2) Fuzzy numbers are convex (i.e., $\left.\mu_{\tilde{M}}(x) \geq \min \left\{\mu_{\tilde{M}}(b), \mu_{\tilde{M}}(a)\right\} \forall a \leq x \leq b\right)$.

(3) The membership function of fuzzy numbers is an upper semi-continuous function.

Several types of fuzzy numbers present in literature, like L-fuzzy numbers, triangular fuzzy numbers, trapezoidal fuzzy numbers, etc.; However, a triangular fuzzy number is used in this study. A triangular fuzzy number $\tilde{M}$ is a special kind of fuzzy number. It is considered as fuzzy variable determined by triplet $(r, s, u)$ such that $(r<s<u)$, with membership function as,

$$
\begin{aligned}
\mu(\tilde{M}) & =\frac{x-r}{s-r}, & & \text { if } r \leq x \leq s \\
& =\frac{u-x}{u-s}, & & \text { if } s \leq x \leq u \\
& =0, & & \text { otherwise. }
\end{aligned}
$$

\subsection{Credibility measure}

In this study, the fuzzy SBM DEA model is approached by credibility measure, and the credibility measure is defined as,

Credibility measure: Consider $\xi$ be a nonempty set with $P\{\xi\}$ be the power set of $\xi$. Liu and Liu [29] defined the credibility set function $\operatorname{Cr}\{$.$\} as a credibility measure if it holds the following conditions:$

(1) $\operatorname{Cr}\{\xi\}=1$,

(2) $\operatorname{Cr}\{Y\} \leq \operatorname{Cr}\{Z\}$ whenever $Y \subset Z \in \xi$, 
(3) $\operatorname{Cr}\{Y\}+\operatorname{Cr}\{Y\}^{C}=1$ for any event $Y \in \xi$,

(4) $\operatorname{Cr}\left\{\cup_{i} Y_{i}\right\}=\operatorname{Sup}_{i} \operatorname{Cr}\left\{Y_{i}\right\}$ for any events $Y_{i}$ with $\operatorname{Sup}_{i} \operatorname{Cr}\left\{Y_{i}\right\}<0.5$.

The triplet $(\xi, P(\xi), C r)$ are called the credibility space.

\subsection{Fuzzy SBM DEA model}

Model (2.1) assumed that all input and output data are exactly known. But, in a real-world problem, this is only an ideal situation that rarely occurs. Therefore, the SBM DEA model is converted into a fuzzy SBM DEA model by assuming the input and output as a fuzzy number. Consider, $i$ th input of the $z$ th DMU be indicated by $\tilde{x}_{i z}$ and the $j$ th output of the $z$ th DMU be indicated by $\tilde{y}_{j z}$, are fuzzy input and output for $\mathrm{DMU}_{z}$, respectively. Then, the fuzzy SBM DEA model with the variable return to scale for $\mathrm{DMU}_{z}$ is given by,

$$
\begin{aligned}
\min \quad \rho_{z}= & \frac{1-\frac{1}{m} \sum_{i=1}^{m} s_{i z}^{-} / \tilde{x}_{i z}}{1+\frac{1}{n} \sum_{j=1}^{n} s_{j z}^{+} / \tilde{y}_{j z}} \\
\text { subject to: } \quad & \sum_{o=1}^{r} \lambda_{o z} \tilde{x}_{i o}+s_{i z}^{-}=t \tilde{x}_{i z} \quad \forall i=1, \cdots, m \\
& \sum_{o=1}^{r} \lambda_{o z} \tilde{y}_{j o}-s_{j z}^{+}=t \tilde{y}_{j z} \quad \forall j=1, \cdots, n \\
& \sum_{o=1}^{r} \lambda_{o}=1 \\
& \lambda_{o z} \geq 0, s_{i z}^{-} \geq 0, s_{j z}^{+} \geq 0, \quad \forall o=1, \cdots, r .
\end{aligned}
$$

The theory of credibility of fuzzy events and chance-constrained programming (CCP) is used in this study to solve the fuzzy SBM DEA model. Wen et al. [44] has given the following results, which are used in solving the process of our fuzzy model.

Theorem 2.1. Consider $\psi_{1}$ and $\psi_{2}$ are two fuzzy variables defined on credibility space $(\xi, P(\xi), C r)$. If $\operatorname{Cr}\left\{\psi_{1}=\right.$ $y\}$ and $\operatorname{Cr}\left\{\psi_{2}=y\right\}$ are quasi concave, then

(1) $\operatorname{Cr}\left\{\psi_{1}+\psi_{2} \leq d\right\} \geq \alpha$ iff $\left(\psi_{1}\right)_{2(1-\alpha)}^{U}+\left(\psi_{2}\right)_{2(1-\alpha)}^{U} \leq d$,

(2) $\operatorname{Cr}\left\{\psi_{1}+\psi_{2} \leq d\right\} \leq \alpha$ iff $\left(\psi_{1}\right)_{2(1-\alpha)}^{U}+\left(\psi_{2}\right)_{2(1-\alpha)}^{U} \geq d$. Here, $0.5 \leq \alpha \leq 1$.

Theorem 2.2. Consider $(\psi)_{\alpha}^{L}$ and $(\psi)_{\alpha}^{U}$ are the lower and upper bounds of $\alpha$-cut of $\psi$, respectively. Then,

(1) if $k \geq 0$, then $(k \psi)_{\alpha}^{U}=k(\psi)_{\alpha}^{U}$ and $(k \psi)_{\alpha}^{L}=k(\psi)_{\alpha}^{L}$,

(2) if $k \leq 0$, then $(k \psi)_{\alpha}^{U}=k(\psi)_{\alpha}^{L}$ and $(k \psi)_{\alpha}^{L}=k(\psi)_{\alpha}^{U}$.

The credibility distribution of triangular fuzzy number $\tilde{M}$ given in (2.2) is defined as,

$$
\begin{aligned}
\operatorname{Cr}(\tilde{M} \leq b) & =0, & & \text { if } r \geq b \\
& =\frac{b-r}{2(s-r)}, & & \text { if } r \leq b \leq s \\
& =\frac{b-2 s+u}{2(u-s)}, & & \text { if } s \leq b \leq u \\
& =1, & & r \leq b . \\
\operatorname{Cr}(\tilde{M} \geq b) & =1, & & \text { if } r \geq b \\
& =\frac{2 s-r-b}{2(s-r)}, & & \text { if } r \leq b \leq s
\end{aligned}
$$




$$
\begin{array}{ll}
=\frac{u-b}{2(u-s)}, & \text { if } s \leq b \leq u \\
=0, & r \leq b .
\end{array}
$$

According to credibility measure, converting fuzzy-chance constraints into their equivalent crisp ones at a particular confidence level $\alpha \geq 0.5$ is as equation (2.6):

$$
\begin{aligned}
& \operatorname{Cr}(\tilde{M} \leq b) \geq \alpha \Longleftrightarrow(2-2 \alpha) s+(2 \alpha-1) u \\
& \operatorname{Cr}(\tilde{M} \geq b) \geq \alpha \Longleftrightarrow(2-2 \alpha) s+(2 \alpha-1) r
\end{aligned}
$$

The SBM DEA model becomes the credibility SBM DEA model with the help of model given by Wen et al. $[44,45]$ as,

$$
\begin{cases}\min f_{z} & \\ \text { subject to: } & \\ \operatorname{Cr}\left\{\frac{1-\frac{1}{m} \sum_{i=1}^{m} s_{i z}^{-} / \tilde{x}_{i z}}{1+\frac{1}{n} \sum_{j=1}^{n} s_{j z}^{+} / \tilde{y}_{j z}} \leq f_{z}\right\} \leq \alpha & \\ \operatorname{Cr}\left\{\sum_{o=1}^{r} \lambda_{o z} \tilde{x}_{i o}+s_{i z}^{-} \leq \tilde{x}_{i z}\right\} \geq \alpha & \forall i=1, \cdots, m \\ \operatorname{Cr}\left\{\sum_{o=1}^{r} \lambda_{o z} \tilde{y}_{j o}-s_{j z}^{+} \geq \tilde{y}_{j z}\right\} \geq \alpha & \forall j=1, \cdots, n \\ \sum_{o=1}^{r} \lambda_{o}=1 & \\ \quad \lambda_{o z} \geq 0, \quad s_{i z}^{-} \geq 0, \quad s_{j z}^{+} \geq 0, & \forall o=1, \cdots, r .\end{cases}
$$

If the membership functions of fuzzy variable are normal and convex. The lower $\alpha$-cut of triangular fuzzy number $\frac{\tilde{a}}{\tilde{b}}$, given as, $\left\{\frac{\tilde{a}}{\tilde{b}}\right\}_{\alpha}^{L}=\tilde{a}_{\alpha}^{L} * 1 /\left(\tilde{b}_{\alpha}^{U}\right)$. The model (2.7) transformed to model (2.8) [32] as follows:

$$
\begin{cases}\min \left\{t-\frac{1}{m} \sum_{i=1}^{m} S_{i z}^{-} / \tilde{x}_{i z}\right\}_{2(1-\alpha)}^{L} & \\ \text { subject to: } & \\ \left\{t+\frac{1}{n} \sum_{j=1}^{n} S_{r z}^{+} / \tilde{y}_{j z}\right\}_{2(1-\alpha)}^{U}=1 & \\ \left\{\sum_{o=1, o \neq z}^{r} \Lambda_{o z} \tilde{x}_{i o}\right\}_{2(1-\alpha)}^{U}+\left\{\Lambda_{o z} \tilde{x}_{i z}\right\}_{2(1-\alpha)}^{L}+S_{i z}^{-} \leq\left\{t \tilde{x}_{i z}\right\}_{2(1-\alpha)}^{L} & \forall i=1, \cdots, m \\ \left\{\sum_{o=1, o \neq z}^{r} \Lambda_{o z} \tilde{y}_{j o}\right\}_{2(1-\alpha)}^{L}+\left\{\Lambda_{o z} \tilde{y}_{j z}\right\}_{2(1-\alpha)}^{U}+S_{j z}^{+} \leq\left\{t \tilde{y}_{j z}\right\}_{2(1-\alpha)}^{U} & \forall j=1, \cdots, n \\ \sum_{o=1}^{r} \Lambda_{o}=t & \forall o=1, \cdots, r .\end{cases}
$$

Here, $S_{i z}^{-}=t s_{i z}^{-}, S_{j z}^{+}=t s_{j z}^{+}, \Lambda_{o z}=t \lambda_{o z}, \Lambda_{o}=t \lambda_{o}$. Model (2.8) is a linear programming model at every $\alpha$, and thus, it can be solved using any software like MATLAB, LINGO, PYTHON, etc. The model (3.2) is used to solve the numerical problem where the input and output slacks is calculated as, $s_{i z}^{-}=S_{i z}^{-} / t$ and $s_{j z}^{+}=S_{j z}^{+} / t$, respectively. The study's primary objective is to do the sensitivity and stability analysis of the model (2.7) and use the analysis to compute the input and output targets for both inefficient and efficient DMUs. The efficient DMUs already performed as the reference set for the inefficient DMUs therefore, the input and output targets for the efficient DMUs are defined as follows:

\subsection{Definitions}

Some important definitions which will be used in the solving process of our fuzzy SBM DEA model. 
Definition 2.3. $\mathrm{DMU}_{z}$ is efficient if $s_{i}^{-*}$ and $s_{j}^{+*}$ are zero for $i=1,2, \cdots, m$ and $j=1,2, \cdots, n$ where $s_{i}^{-*}$ and $s_{j}^{+*}$ are optimal solutions of model (2.1).

Definition 2.4 ( $\alpha$-efficiency). $\operatorname{DMU}_{z}$ is $\alpha$-efficient if $s_{i}^{-*}(\alpha)$ and $s_{j}^{+*}(\alpha)$ are zero for $i=1,2, \cdots, m$ and $j=$ $1,2, \cdots, n$ where $s_{i}^{-*}(\alpha)$ and $s_{j}^{+*}(\alpha)$ are optimal solutions of model (2.6) at fixed value of $\alpha$.

Definition 2.5 (Target input). The lower bound for the input for which inefficient DMUs will becomes efficient.

Definition 2.6 (Target output). The upper bound for the output for which inefficient DMUs will becomes efficient.

Definition 2.7 (Input target for efficient DMUs). Maximum output values for which efficient DMUs will remain efficient.

Definition 2.8 (Output target for efficient DMUs). Minimum output values for which efficient DMUs will remain efficient.

\section{SENSITIVITY ANALYSIS}

Sensitivity and stability analysis has been done in the next subsections for the fuzzy SBM DEA models. Since DEA is data-based, it is fascinating to assess possible input and output changes (data perturbation) of a DMU such that its obtained efficiency classification does not change.

\subsection{Sensitivity analysis of inefficient DMUs}

In this section, the two theorems are proposed based on which the slacks for the inefficient DMUs are calculated.

Theorem 3.1. If $\mathrm{DMU}_{z}$ is $\alpha$-inefficient, then the optimal solution satisfying $\lambda_{z}^{*}(\alpha)=0$.

Proof. For fixed $\alpha$, the optimal solution of model $(2.5)$ is $\left(\lambda_{j}^{*}, s_{i}^{-*}, s_{j}^{+*}\right)$. Consider $\lambda_{z}^{*}>0$ then there exists at least one $s_{i}^{-*}>0$ or $s_{j}^{+*}>0$ because $\mathrm{DMU}_{z}$ is not efficient. Assume $s_{i}^{-*}>0$. If $\lambda_{z}^{*}=1$, then $\operatorname{Cr}\left\{\tilde{x}_{i o}+s_{i z}^{-}=\tilde{x}_{i z}\right\}$ is zero. But, $\operatorname{Cr}\left\{\tilde{x}_{i o}+s_{i z}^{-}=\tilde{x}_{i z}\right\} \geq \alpha$, which implies that $\lambda_{z}^{*} \neq 1$. This implies $0<\lambda_{z}^{*}<1$ and it can be obtained that,

$$
\begin{cases}\operatorname{Cr}\left\{\sum_{o=1}^{r} \lambda_{o}^{*} \tilde{x}_{i o}+s_{i z}^{-*} \leq \tilde{x}_{i z}\right\} \geq \alpha & \forall i=1, \cdots, m \\ \operatorname{Cr}\left\{\sum_{o=1, o \neq z}^{r} \lambda_{o}^{*} \tilde{x}_{i o}+\lambda_{z}^{*} \tilde{x}_{i z}+s_{i z}^{-*} \leq \tilde{x}_{i z}\right\} \geq \alpha & \forall i=1, \cdots, m \\ \operatorname{Cr}\left\{\frac{\left(1-\lambda_{z}^{*}\right) \sum_{o=1, o \neq z}^{r} \lambda_{o}^{*} \tilde{x}_{i o}}{\left(1-\lambda_{z}^{*}\right)} \leq\left(1-\lambda_{z}^{*}\right) \tilde{x}_{i z}-s_{i z}^{-*}\right\} \geq \alpha & \forall i=1, \cdots, m \\ \operatorname{Cr}\left\{\frac{\sum_{o=1, o \neq z}^{r} \lambda_{o}^{*} \tilde{x}_{i o}}{\left(1-\lambda_{z}^{*}\right)} \leq \frac{\left(1-\lambda_{z}^{*}\right) \tilde{x}_{i z}-s_{i z}^{-*}}{\left(1-\lambda_{z}^{*}\right)}\right\} \geq \alpha & \forall i=1, \cdots, m \\ \operatorname{Cr}\left\{\frac{\sum_{o=1, o \neq z}^{r} \lambda_{o}^{*} \tilde{x}_{i o}}{\left(1-\lambda_{z}^{*}\right)} \leq \tilde{x}_{i z}-\frac{s_{i z}^{-*}}{\left(1-\lambda_{z}^{*}\right)}\right\} \geq \alpha & \forall i=1, \cdots, m .\end{cases}
$$

Thus, $\left\{\frac{\lambda_{1}^{*}}{\sum_{o=1, o \neq z}^{r} \lambda_{o}^{*}}, \cdots, \frac{\lambda_{2}^{*}}{\sum_{o=1, o \neq z}^{r} \lambda_{o}^{*}}, 0, \frac{\lambda_{r-1}^{*}}{\sum_{o=1, o \neq z}^{r} \lambda_{o}^{*}}, \frac{\lambda_{r}^{*}}{\sum_{o=1, o \neq z}^{r} \lambda_{o}^{*}}\right\}$ is also a feasible solution and the objective value is $\frac{f_{z}}{1-\lambda_{z}^{*}}>f_{z}$, which leads to a contradiction. Thus, $\lambda_{z}^{*}(\alpha)=0$.

Theorem $3.2([44])$. An $\alpha$-inefficient $\mathrm{DMU}_{z}$ becomes $\alpha$-efficient if $\left(x_{z}, y_{z}\right)=\left(x_{z}-s_{i}^{-*}(\alpha), y_{z}+s_{j}^{+*}(\alpha)\right)$, in which $s_{i}^{-*}(\alpha)$ and $s_{j}^{+*}(\alpha)$ are optimal solutions of model (2.5) at fixed value of $\alpha$. 


\subsection{Stability analysis of efficient DMUs}

Theorem 3.3. An $\alpha$-efficient $\mathrm{DMU}_{z}$ stays $\alpha$-efficient if $\left(x_{z}, y_{z}\right)=\left(x_{z}+t_{i z}^{+*}(\alpha), y_{z}-t_{j z}^{-*}(\alpha)\right)$, in which $t_{i z}^{+*}(\alpha)$ and $t_{j z}^{-*}(\alpha)$ are optimal solutions of model (2.7) at $\alpha$.

Proof. A new fuzzy model (3.1) is proposed to do the stability analysis for the efficient DMUs.

$$
\begin{cases}\min f_{z} & \\ \text { subject to: } & \\ \operatorname{Cr}\left\{\frac{1+\frac{1}{m} \sum_{i=1}^{m} t_{i z}^{+} / \tilde{x}_{i z}}{1-\frac{1}{n} \sum_{j=1}^{n} t_{j z}^{-} / \tilde{y}_{j z}} \leq f_{z}\right\} \leq \alpha & \\ \operatorname{Cr}\left\{\sum_{o=1, o \neq z}^{r} \lambda_{o} \tilde{x}_{i o}-t_{i z}^{+} \leq \tilde{x}_{i z}\right\} \geq \alpha & \forall i=1, \cdots, m \\ \operatorname{Cr}\left\{\sum_{o=1, o \neq z}^{r} \lambda_{o} \tilde{y}_{j o}+t_{j z}^{-} \geq \tilde{y}_{j z}\right\} \geq \alpha & \forall j=1, \cdots, n \\ \sum_{o=1}^{r} \lambda_{o}=1 & \\ \quad \lambda_{o} \geq 0, t_{i z}^{+} \geq 0, \quad t_{j z}^{-} \geq 0 & \forall o=1, \cdots, r .\end{cases}
$$

Convert $\left(\tilde{x}_{z}, \tilde{y}_{z}\right)$ into the $\left(\tilde{x}_{z}+t_{i z}^{+*}, \tilde{y}_{z}-t_{j z}^{-*}\right)$ in the model (3.1). Then, the model (3.1) is equivalent to model (3.2).

$$
\begin{cases}\min f_{z} & \\ \text { subject to: } & \\ \operatorname{Cr}\left\{\frac{1+\frac{1}{m} \sum_{i=1}^{m} s_{i}^{-} /\left(\tilde{x}_{i z}+t_{i z}^{+}\right)}{1-\frac{1}{n} \sum_{j=1}^{n} s_{r}^{+} /\left(\tilde{y}_{j z}-t_{j z}^{-}\right)} \leq f_{z}\right\} \leq \alpha & \\ \operatorname{Cr}\left\{\sum_{o=1, o \neq z}^{r} \lambda_{o} \tilde{x}_{i o}+\lambda_{z}\left(\tilde{x}_{i z}+t_{i z}^{+}\right)+s_{i z}^{-} \leq \tilde{x}_{i z}+t_{i z}^{+}\right\} \geq \alpha & \forall i=1, \cdots, m \\ \operatorname{Cr}\left\{\sum_{o=1, o \neq z}^{r} \lambda_{o} \tilde{y}_{j o}+\lambda_{z}\left(\tilde{y}_{o z}-t_{j z}^{-}\right)-s_{j z}^{+} \geq \tilde{y}_{j z}-t_{j z}^{-}\right\} \geq \alpha & \forall j=1, \cdots, n \\ \sum_{o=1}^{r} \lambda_{o}=1 & \\ \quad \lambda_{o} \geq 0, \quad s_{i z}^{-} \geq 0, \quad s_{j z}^{+} \geq 0 & \forall o=1, \cdots, r .\end{cases}
$$

Assume that the $\mathrm{DMU}_{z}$ is inefficient therefore, $\lambda_{z}^{*}=0$ (Thm. 3.1). Then, the model (3.2) transformed to model (3.3) as,

$$
\begin{cases}\min f_{z} & \\ \text { subject to : } & \\ \operatorname{Cr}\left\{\frac{1+\frac{1}{m} \sum_{i=1}^{m} s_{i}^{-} /\left(\tilde{x}_{i z}+t_{i z}^{+}\right)}{1-\frac{1}{n} \sum_{j=1}^{n} s_{r}^{+} /\left(\tilde{y}_{j z}-t_{j z}^{-}\right)} \leq f_{z}\right\} \leq \alpha & \\ \operatorname{Cr}\left\{\sum_{o=1, o \neq z}^{r} \lambda_{o} \tilde{x}_{i o}-t_{i z}^{+*}+s_{i}^{-} \leq \tilde{x}_{i z}\right\} \geq \alpha & \forall i=1, \cdots, m \\ \operatorname{Cr}\left\{\sum_{o=1, o \neq z}^{r} \lambda_{o} \tilde{y}_{j o}+t_{j z}^{-*}-s_{j}^{+} \geq \tilde{y}_{j z}\right\} \geq \alpha & \forall j=1, \cdots, n \\ \quad \sum_{o=1}^{r} \lambda_{o}=1 & \\ \quad \lambda_{o} \geq 0, \quad s_{i z}^{-} \geq 0, \quad s_{j z}^{+} \geq 0 & \end{cases}
$$


TABle 2. Guo and Tanaka's [16] fuzzy data.

\begin{tabular}{llllll}
\hline \hline DMUs & 1 & 2 & 3 & 4 & 5 \\
\hline Input $_{1}$ & $(3.5,4.0 .4 .5)$ & $(2.9,2.9,2.9)$ & $(4.4,4.9,5.4)$ & $(3.4,4.1,4.8)$ & $(5.9,6.5,7.1)$ \\
Input $_{2}$ & $(1.9,2.1,2.3)$ & $(1.4,1.5,1.6)$ & $(2.2,2.6,3.0)$ & $(2.1,2.3,2.5)$ & $(3.6,4.1,4.6)$ \\
Output $_{1}$ & $(2.4,2.6,2.8)$ & $(2.2,2.2,2.2)$ & $(2.7,3.2,3.7)$ & $(2.5,2.9,3.3)$ & $(4.4,5.1,5.8)$ \\
Output $_{2}$ & $(3.8,4.1,4.4)$ & $(3.3,3.5,3.7)$ & $(4.3,5.1,5.9)$ & $(5.5,5.7,5.9)$ & $(6.5,7.4,8.3)$ \\
\hline
\end{tabular}

TABLE 3. Comparison between the sensitivity analysis results of inefficient \& efficient DMUs.

\begin{tabular}{lllllllllll}
\hline \hline \multirow{2}{*}{ STUs } & \multicolumn{4}{c}{ Wen et al. [45] } & \multicolumn{5}{c}{ Proposed approach } \\
\cline { 2 - 11 } & $s_{1}^{+*}$ & $s_{2}^{+*}$ & $s_{1}^{-*}$ & $s_{2}^{-*}$ & Efficiency & $s_{1}^{+*}$ & $s_{2}^{+*}$ & $s_{1}^{-*}$ & $s_{2}^{-*}$ & Efficiency \\
\hline DMU $_{1}$ & 0.15 & 0.00 & 0.00 & 0.60 & Inefficient & 0.5227 & 0.2710 & 0 & 0.5331 & 0.6422 \\
DMU $_{2}$ & 1.20 & 0.66 & 0.00 & 0.00 & Efficient & 0.00 & 0.00 & 0.06 & 0.00 & 1.0269 \\
DMU $_{3}$ & 0.47 & 0.05 & 0.00 & 0.83 & Inefficient & 0.6490 & 0.3381 & 0 & 0.5445 & 0.6494 \\
DMU $_{4}$ & 0.00 & 0.17 & 0.00 & 1.01 & Efficient & 0.00 & 0.00 & 0.00 & 0.6882 & 1.1395 \\
DMU $_{5}$ & 0.00 & 0.00 & 2.42 & 1.92 & Efficient & 1.42 & 1.62 & 0.00 & 0.00 & 2.104 \\
\hline
\end{tabular}

The optimal solution of model (3.3) is feasible solution of model (3.1). Hence, $t_{i z}^{+*}-s_{i}^{-*} \geq t_{i z}^{+*}$ and $t_{j z}^{-*}-s_{j}^{+*} \geq t_{j z}^{-*}$, which means that $s_{i}^{-*}=0$ and $s_{j}^{+*}=0, \forall i=1,2, \cdots, m$ and $j=1,2, \cdots, n$. Which is contradiction.

$\Longrightarrow \mathrm{DMU}_{z}$ stays $\alpha$-efficient if $\left(x_{z}, y_{z}\right)=\left(x_{z}+t_{i z}^{+*}(\alpha), y_{z}-t_{j z}^{-*}(\alpha)\right)$, in which $t_{i z}^{+*}(\alpha)$ and $t_{j z}^{-*}(\alpha)$ are optimal solutions of model (3.3) at fixed value of $\alpha$. Thus, model (3.1) will compute the efficient targets for the efficient DMUs at different values of alphas.

\section{NumERICAL ILLUSTRATION}

A numerical experiment is depicted here for the comparison between Wen et al. [45] and the proposed study. The data used for the comparison is fuzzy, which has been taken from Guo and Tanaka's [16] study. The data has two inputs and two outputs which are fuzzy triangular numbers. Table 2 displays the data for all DMUs.

The relative efficiency and slacks have been computed at $\alpha=0.6$. Table 3 shows the comparison between the results obtained by Wen et al. [45] and the proposed study. It is clear from Table 3 that from both the methods, $\mathrm{DMU}_{1}, \mathrm{DMU}_{3}$ are inefficient and $\mathrm{DMU}_{2}, \mathrm{DMU}_{4}$, and $\mathrm{DMU}_{5}$ are efficient. The slacks for both efficient and inefficient DMU are approximately comparable. But, there is a crucial difference between both studies; the proposed approach can compute the efficiency value while Wen et al. [45] is only capable of determining whether the DMUs are efficient or inefficient. For efficiency score, a dual form is needed for Wen et al. [45]. In addition, the applicability of the proposed research in the transport sector is illustrated in the following section.

\section{An APPLiCATiOn in TRANSPORTATiON}

DEA has been used in many applications of transportation, and so we selected input and output variables based on previous research; for the full list of applications, see [33]. The transport data of State Transport Undertakings (STUs) [4] has been collected, and the proposed model has been applied to calculate the relative efficiency and the stable regions for efficient STUs. The transport sector has been chosen because the transport sector's development directly contributes to the country's economic growth as it links with exporting commodities, production, etc. STUs are one of the primary sources for people's mobility in a developing country like INDIA. The respective state government controls the STUs. The STUs allow the public to use non-profitable 
social services. Therefore, it is imperative to screen their relative performance to improve output efficiency consistently. The data of STUs for the annual year 2015-2016 of 30 STUs has been collected. Various factors can affect transportation, but the study considers four important criteria: Fleet Size, Total Staff, Fuel Consumption (inputs), and Passenger kilometers (output).

- Fleet size. The total number of buses on-road in an STU; this attribute is defined as capital input.

- Total staff. Total employees who worked in an STU are the entire staff; this attribute is set as a labor input.

- Effective kilometer per litre. The fuel consumed, which is measured by dividing the total effective kilometer per liter kilometer; this attribute is defined as a material input.

- Passenger-kilometers. It is a measure of service utilization, representing the overall sum of the distances ridden by each passenger. This attribute is computed by taking the sum of the passenger load times with the gap between individual bus stops.

All the data is collected from the report published by the Central Institute of Road Transport (CIRT) for the year 2015-2016. But still, some data is missing in this publication. The fuzzy numbers have been used as a replacement for the missing data with the help of the following algorithm:

- Clustering of data into 6 clusters.

- Assumed missing value as a triangular fuzzy number.

- The triangular fuzzy number is, (Minimum value from the cluster from the respective cluster from which the missing value belongs, Mean of Minimum and Maximum, Maximum value from the cluster from the respective cluster from which the missing value belongs).

Tables 4 and 6 show the relative efficiencies of all the STUs at a different level of credibility by the proposed fuzzy SBM DEA model using MATLAB R2019b.

\subsection{Sensitivity analysis for inefficient STUs}

The relative efficiency of all the STUs is calculated at credibility level 0.5 and 1 . After that, the input and output slacks values are calculated using the model (2.6) for all the inefficient STUs.

It is evident from Table 4 that the total of 18 STUs out of 30 STUs are credibilisitically inefficient STUs. The input and output targets are also can be computed from table. For example, STU SBSTC is credibilisitically inefficient at 0.5 and remained inefficient for the following region: $\left(\hat{x}_{A 1}, \hat{x}_{A 2}, \hat{x}_{A 3}, \hat{y}_{A 1}\right)=\left(\tilde{x}_{A 1}-r_{x 1}, \tilde{x}_{A 2}-\right.$ $\left.r_{x 2}, \tilde{x}_{A 13}-r_{x 3}, \tilde{y}_{A 1}+r_{y 1}\right)$, where $0 \leq r_{x 1}<336.6735,0 \leq r_{x 2}<121.5703, r_{x 3}=31.5164$ and $r_{y 1}=0$. The STU SBSTC becomes efficient when both inputs and outputs reach simultaneously at their target values. Similarly, the target inputs and target outputs for the other STUs is also calculated. Similarly, the target values for both inputs and outputs at different credibility level can be calculated. The efficient targets give an excellent observation about the efficiency improvements to the transport's operator. They can follow from the obtained results which variables influence most the efficiency and how can they improve it by minimum effort. Operators might not control some variables like fleet size, but they can enhance the other attributes. The proposed study has the characteristic that it can simultaneously compute the relative efficiency and efficient target, which is helpful for fast computations (Tab. 5).

\subsection{Stability analysis for efficient STUs}

The stability analysis results for the efficient DMUs for which the efficient DMUs remains efficient is calculated using model (2.8) and given in Table 6.

From Table 7, STU TNSTC-VPM remained efficient for the interval, $\left(\hat{x}_{B 1}, \hat{x}_{B 2}, \hat{x}_{B 3}, \hat{y}_{B 1}\right)=\left(\tilde{x}_{B 1}+t_{x 1}, \tilde{x}_{B 2}+\right.$ $\left.t_{x 2}, \tilde{x}_{B 3}+t_{x 3}, \tilde{y}_{B 1}-t_{y 1}\right)$, where $t_{x 1}=231.96, t_{x 2}=0, t_{x 3}=96.92$ and $t_{y 1}=0$.

Similarly, the stability analysis results for efficient STUs for which the STUs remains credibilisitically efficient can also be calculated. 
TABLE 4. Slacks for inefficient DMUs.

\begin{tabular}{|c|c|c|c|c|c|c|c|c|c|c|}
\hline \multirow[t]{2}{*}{ STUs } & \multicolumn{5}{|c|}{0.5} & \multicolumn{5}{|c|}{1} \\
\hline & Efficiency & $s_{1}^{-}$ & $s_{2}^{-}$ & $s_{3}^{-*}$ & $s_{1}^{+*}$ & Efficiency & $s_{1}^{-*}$ & $s_{2}^{-*}$ & $s_{3}^{-*}$ & $s_{1}^{+*}$ \\
\hline KDTC & 0.5829 & 342.3833 & 705.531 & 23.821 & 0 & 1 & 0 & 0 & 0 & 0 \\
\hline SBSTC & 0.7134 & 336.6735 & 121.5703 & 31.5164 & 0 & 0.7134 & 336.6735 & 121.5703 & 31.5164 & 0 \\
\hline OSRTC & 1 & 0 & 0 & 0 & 0 & 1 & 0 & 0 & 0 & 0 \\
\hline TMTU & 0.5177 & 198.1867 & 968.5598 & 20.9996 & 0 & 0.5177 & 198.1867 & 968.5598 & 20.9996 & 0 \\
\hline KMTU & 1 & 0 & 0 & 0 & 0 & 1 & 0 & 0 & 0 & 0 \\
\hline NMMT & 0.5469 & 181.2749 & 1083.3143 & 37.5339 & 0 & 0.5469 & 181.2749 & 1083.3143 & 37.5339 & 0 \\
\hline APSRTC & 1 & 0 & 0 & 0 & 0 & 1 & 0 & 0 & 0 & 0 \\
\hline TSRTC & 0.8613 & 2180.3771 & 10250.6604 & 101.0549 & 0 & 0.8613 & 2180.3771 & 10250.6604 & 101.0549 & 0 \\
\hline MSRTC & 1 & 0 & 0 & 0 & 0 & 1 & 0 & 0 & 0 & 0 \\
\hline RSRTC & 0.7847 & 1424.3288 & 1338.4327 & 310.1936 & 0 & 0.7847 & 1424.3288 & 1338.4327 & 310.1936 & 0 \\
\hline NWKnRTC & 0.5975 & 2098.6161 & 9364.5317 & 413.9542 & 0 & 0.5975 & 2098.6161 & 9364.5317 & 413.9542 & 0 \\
\hline TNSTC-KUM & 1 & 0 & 0 & 0 & 0 & 1 & 0 & 0 & 0 & 0 \\
\hline TNSTC-VPM & 1 & 0 & 0 & 0 & 0 & 1 & 0 & 0 & 0 & 0 \\
\hline NEKnRTC & 0.6117 & 2092.6197 & 7615.3933 & NW19 298.6089 & 0 & 0.6117 & 2092.6197 & 7615.3933 & 298.6089 & 0 \\
\hline SETC-TN & 1 & 0 & 0 & 0 & 0 & 1 & 0 & 0 & 0 & 0 \\
\hline ASMSTC & 0.3387 & 2679.3235 & 1847.0076 & 146.3528 & 0 & 0.3387 & 2679.3235 & 1847.0076 & 146.3528 & 0 \\
\hline HRTC & 0.3854 & 1920.7207 & 4852.5578 & 305.3603 & 0 & 0.3854 & 1920.7207 & 4852.5578 & 305.3603 & 0 \\
\hline UTC & 1 & 0 & 0 & 0 & 0 & 1 & 0 & 0 & 0 & 0 \\
\hline PMPML & 0.4181 & 1372.4782 & 5945.2721 & 171.0854 & 0 & 0.4181 & 1372.4782 & 5945.2721 & 171.0854 & 0 \\
\hline GSRTC & 0.7492 & 2432.9731 & 8218.0189 & 458.2274 & 0 & 0.7492 & 2432.9731 & 8218.0189 & 458.2274 & 0 \\
\hline UPSRTC & 1 & 0 & 0 & 0 & 0 & 1 & 0 & 0 & 0 & 0 \\
\hline KnSRTC & 0.8193 & 2139.8912 & 3339.5699 & 381.5114 & 0 & 0.8193 & 2139.8912 & 3339.5699 & 381.5114 & 0 \\
\hline TNSTC-MDU & 1 & 0 & 0 & 0 & 0 & 1 & 0 & 0 & 0 & 0 \\
\hline TNSTC-SLM & 1 & 0 & 0 & 0 & 0 & 1 & 0 & 0 & 0 & 0 \\
\hline DTC & 0.4639 & 2708.0051 & 20435.6894 & 262.8264 & 0 & 0.4792 & 1914.9247 & 16123.5977 & 0 & 52838.7525 \\
\hline MTC & 0.7616 & 1014.131 & 9751.5489 & 47.9188 & 0 & 0.7616 & 1014.131 & 9751.5489 & 47.9188 & 0 \\
\hline KSRTC & 0.8296 & 1747.6148 & 1456.9822 & 173.7345 & 0 & 1 & 0 & 0 & 0 & 0 \\
\hline BMTC & 0.542 & 3446.1061 & 18893.3766 & 365.1995 & 0 & 0.542 & 3446.1061 & 18893.3766 & 365.1995 & 0 \\
\hline
\end{tabular}

TABLE 5. Lower bounds for inputs and upper bounds for outputs for STU SBSTC.

\begin{tabular}{ll}
\hline \hline Fleet size & $(661-336.6735,661-336.6735,661-336.6735=324.3265,324.3265,324.3265)$ \\
Total staff & $(2117-121,2117-121,2117-121=1996,1996,1996)$ \\
Effective KMPL & $(107.57-31.5164,107.57-31.5164,107.57-31.5164=76.0536,76.0536,76.0536)$ \\
Passenger kilometers & $(16626.34+0,16626.34+0,16626.34+0+1=16626.34,16626.34,16626.34)$ \\
\hline
\end{tabular}

TABLE 6. Slacks for efficient DMUs.

\begin{tabular}{|c|c|c|c|c|c|c|c|c|}
\hline STUs & & & 0.5 & & & & 1 & \\
\hline Efficiency & $t_{1}^{+*}$ & $t_{2}^{+*}$ & $t_{3}^{-*}$ & $t_{1}^{-*}$ & $t_{1}^{+*}$ & $t_{2}^{+*}$ & $t_{3}^{-*}$ & $t_{1}^{-*}$ \\
\hline OSRTC & 1.0026 & 0 & 12.6082 & 0 & 1.0026 & 0 & 12.6082 & 0 \\
\hline KMTU & 2.3824 & 224 & 1105 & 28.11 & 2.3824 & 224 & 1105 & 28.11 \\
\hline APSRTC & 1.1682 & 1647.8549 & 16080.9807 & 330.4939 & 1.1714 & 1647.8549 & 16660.2367 & 330.4939 \\
\hline TNSTC-KUM & 1.0487 & 231.96 & 0 & 96.92 & 1.0487 & 231.96 & 0 & 96.92 \\
\hline TNSTC-VPM & 1.0203 & 17.21 & 1281.1 & 0 & 1.0203 & 17.21 & 1281.1 & 0 \\
\hline
\end{tabular}


TABLE 7. Stability analysis for efficient STU TNSTC-VPM.

\begin{tabular}{ll}
\hline \hline Fleet size & $(3680+231.96,3680+231.96,3680+231.96=3911.96,3911.96,3911.96)$ \\
Total staff & $(22741,22741,22741)$ \\
Effective & $(1151.71+96.92,1151.71+96.92,1151.71+96.92)=(1248.63,1248.63,1248.63)$ \\
KMPL & \\
Passenger & $(269338,269338,269338)$ \\
kilometers & \\
(in lakhs) & \\
\hline
\end{tabular}

\section{Managerial implications}

One of the edges of the proposed approach other than the more complicated approaches is that it is not limited to efficiency computation. It focuses on the input and output improvements that are called slacks in the DEA language. These improvements are also expressed as efficient targets and are helpful to managers. Most existing fuzzy DEA approaches, especially those that use multiplier models, do not provide efficiency. Thus, the goals to their managerial utility are limited. Various methods to solve fuzzy SBM DEA models from which $\alpha$-cut approach, possibility approach, and credibility approach are the most often used techniques. The relative efficiency from the $\alpha$-cut approach gives two different values (lower bound and upper bound) at different values of $\alpha$. Thus, the input and output targets will also have been two different values. It becomes complex to implement these obtained results in real life for the managers. On the other hand, the possibility and credibility approach will lead only to one relative efficiency. The self-dual property of the credibility approach makes it more significant and can be useful in real-life problems. The study proposed how the fuzzy SBM DEA model can calculate input and output targets for both efficient and inefficient firms under the credibility approach. The applicability of the study to the transport problem is also shown. The data of Indian STUs has been collected for the year 2015-2016. There has been some data missing for some STUs. Fuzzy numbers then replace the missing data. 18 inefficient and 12 efficient STUs out of 30 STUs have been obtained after computing the relative efficiency from the fuzzy SBM DEA model. The input and output targets are also computed for both efficient and inefficient STUs. The proposed study can compute both relative efficiency and input and output targets under a fuzzy environment using the credibility approach.

\section{Conclusions}

The study proposed a novel approach to calculate the target inputs and target outputs for inefficient and efficient DMUs using a crediblistic approach. The credibility approach, which manages human conviction numerically, has been used to compute these targets. The proposed method simultaneously computes the relative efficiency of DMUs and targets with simple computation under fuzzy conditions. A comparison between Wen et al. [45]'s approach and the proposed method is presented in the Numerical illustration section. The results are comparable, but the simultaneous calculation of efficiency and efficient targets provides sovereignty to the proposed study. The applicability of the proposed approach has been shown in the transport sector for realworld application. For this, data from Indian STUs have been collected from the CIRT report 2015-2016. Some of the data is missing in the CIRT report due to unknown reasons. The fuzzy numbers are used to fulfill the missing data, and then relative efficiency and efficient targets are calculated. A total of 12 STUs out of 30 are credibilisitically efficient at all credibility levels. Some of the STUs are credibilisitically inefficient at credibility level 0.5 but credibilisitically efficient at one, e.g., KDTC. The slacks are shown in Tables 1 and 3 for the inefficient and efficient DMUs, respectively. These slacks are helpful in the calculation of fuzzy input and output targets at different credibility levels. The study proposed sensitivity and stability analysis for the fuzzy SBM DEA model. The number of efficient DMUs is more than one, and the ranking of these DMUs can be difficult. 
Stability analysis of efficient DMUs can be a help to rank efficient DMUs. The hierarchy of efficient DMUs using the fuzzy SBM DEA model under the credibility approach could be the future of this research.

Acknowledgements. The authors thank DST for providing partial support under FIST grant SR/FST/MSI-090/2013(C) and CSIR for providing financial assistance under JRF programme (Award No.: 09/719/(0079)/2017-EMR-I.

\section{REFERENCES}

[1] S. Agarwal, Efficiency measure by fuzzy data envelopment analysis model. Fuzzy Inf. Eng. 16 (2014) 59-70.

[2] S. Agarwal, SBM data envelopment analysis in fuzzy environment. Math. Sci. Int. Res. J. 3 (2014) $478-484$.

[3] S. Agarwal, Fuzzy slack based measure of data envelopment analysis: a possibility approach. In: Proceedings of the Third International Conference on Soft Computing for Problem Solving (2014) 733-740.

[4] S. Agarwal, S.P. Yadav and S.P. Singh, DEA based estimation of the technical efficiency of state transport undertakings in India. Opsearch 47 (2010) 216-230.

[5] M. Arana-Jiménez, M.C. Sánchez-Gil and S. Lozano, A fuzzy DEA slacks-based approach. J. Comput. Appl. Math. (2020) 113180.

[6] M. Arana-Jimenez, M.C. Sánchez-Gil and S. Lozano, Efficiency assessment and target setting using a fully fuzzy DEA approach. Int. J. Fuzzy Syst. 22 (2020) 1056-1072.

[7] R.D. Baker, Maximum likelihood, consistency and DEA statistical foundations. Manage. Sci. 39 (1993) $1265-1273$.

[8] A. Charnes and W.W. Cooper, Chance-constrained programming. Manage. Sci. 6 (1959) 73-79.

[9] A. Charnes and L. Neralić, Sensitivity analysis of the additive model in data envelopment analysis. Eur. J. Oper. Res. 48 (1990) 332-341.

[10] A. Charnes, W.W. Cooper and E. Rhodes, Measuring the efficiency of decision making units. Eur. J. Oper. Res. 2 (1978) 429-444.

[11] A. Charnes, W.W. Cooper, B. Golany, L. Seiford and J. Stutz, Foundations of data envelopment analysis for Pareto-Koopmans efficient empirical production functions. J. Econometrics 30 (1985) 91-107.

[12] W.W. Cooper, L.M. Seiford and K. Tone, Data envelopment analysis. In: Handbook on Data Envelopment Analysis (2000) $1-40$.

[13] A. Emrouznejad and A. Mustafa, Fuzzy data envelopment analysis: a discrete approach. Expert Syst. App. 39 (2012) $2263-2269$.

[14] A. Emrouznejad and M. Tavana, Performance Measurement with Fuzzy Data Envelopment Analysis. Springer (2014).

[15] A. Emrouznejad and G.L. Yang, A survey and analysis of the first 40 years of scholarly literature in DEA: 1978-2016. Soc.-Econ. Planning Sci. 61 (2018) 4-8.

[16] P. Guo and H. Tanaka, Fuzzy DEA: a perceptual evaluation method. Fuzzy Sets Syst. 119 (2001) 149-160.

[17] P. Gupta, M.K. Mehlawat, A. Kumar, S. Yadav and A. Aggarwal, A credibilistic fuzzy DEA approach for portfolio efficiency evaluation and rebalancing toward benchmark portfolios using positive and negative returns. Int. J. Fuzzy Syst. 22 (2020) $824-843$.

[18] C. Heydari, H. Omrani and R. Taghizadeh, A fully fuzzy network DEA-Range Adjusted Measure model for evaluating airlines efficiency: a case of Iran. J. Air Transp. Manage. 89 (2020) 101923.

[19] G.R. Jahanshahloo, M. Soleimani-Damaneh and E. Nasrabadi, Measure of efficiency in DEA with fuzzy input-output levels: a methodology for assessing, ranking and imposing of weights restrictions. Appl. Math. Comput. 156 (2004) $175-187$.

[20] G.R. Jahanshahloo, F. Hosseinzadeh, N. Shoja, M. Sanei and G. Tohidi, Sensitivity and stability analysis in DEA. Appl. Math. Comput. 169 (2005) 897-904.

[21] D. Kahneman and A. Tversky, Prospect theory: an analysis of decision under risk. Econometrics 47 (1979) $263-291$.

[22] C. Kao, and S.T. Liu, Fuzzy efficiency measures in data envelopment analysis. Fuzzy Sets Syst. 113 (2000) $427-437$.

[23] M.Z.A. Langroudi, A. Emrouznejad, A. Mustafa and J. Ignatius, Type-2 TOPSIS: a group decision problem when ideal values are not extreme endpoints. Group Decision Negotiation 22 (2013) 851-866.

[24] S. Lertworasirikul, S.C. Fang, J.A. Joines and H.L. Nuttle, Fuzzy data envelopment analysis (DEA): a possibility approach. Fuzzy Sets Syst. 139 (2003) 379-394.

[25] S. Lertworasirikul, S.C. Fang, J. Joines and H. Nuttle, Fuzzy data envelopment analysis: a credibility approach. Fuzzy Sets Based Heuristics Optim. (2003) 141-158.

[26] B. Liu, Uncertainty Theory. Springer, Berlin, Heidelberg (2007) 205-234.

[27] B. Liu, Some research problems in uncertainty theory. J. Uncertain Syst. 3 (2009) 3-10.

[28] B. Liu, Uncertainty Theory. Springer, Berlin, Heidelberg (2010) 1-79.

[29] B. Liu and Y.K. Liu, Expected value of fuzzy variable and fuzzy expected value models. IEEE Trans. Fuzzy Syst. 10 (2002) $445-450$.

[30] F.H. Lotfi, M.A. Jondabeh and M. Faizrahnemoon, Senstivity analysis in fuzzy environment. Appl. Math. Sci. 4 (2010) 16351646 .

[31] C.K. Lovell, Measuring the macroeconomic performance of the Taiwanese economy. Int. J. Prod. Econ. 39 (1995) 165-178.

[32] D. Mahla and S. Agarwal, A credibility approach on fuzzy Slacks-Based Measure (SBM) model (2020). DOI: 10.22111/ijfs.2020.31572.5443 (In Press). 
[33] R. Mahmoudi, A. Emrouznejad, S.N. Shetab-Boushehri and S.R. Hejazi, The origins, development and future directions of data envelopment analysis approach in transportation systems. Soc.-Econ. Plann. Sci. 69 (2020) 100672.

[34] L. Neralić and R.E. Wendell, Sensitivity in data envelopment analysis using an approximate inverse matrix. J. Oper. Res. Soc. 55 (2004) 1187-1193.

[35] L. Neralić and R.E. Wendell, Enlarging the radius of stability and stability regions in Data Envelopment Analysis. Eur. J. Oper. Res. 278 (2019) 430-441.

[36] O.B. Olesen and N.C. Petersen, Chance constrained efficiency evaluation. Manage. Sci. 41 (1995) $442-457$.

[37] S. Saati and A. Memariani, SBM model with fuzzy input-output levels in DEA. Aust. J. Basic Appl. Sci. 3 (2009) $352-357$.

[38] M. Sanei, N. Noori and H. Saleh, Sensitivity analysis with fuzzy data in DEA. Appl. Math. Sci. 3 (2009) 1235-1241.

[39] L.M. Seiford and J. Zhu, Sensitivity and stability of the classifications of returns to scale in data envelopment analysis. J. Prod. Anal. 12 (1999) 55-75.

[40] J.K. Sengupta, Efficiency measurement in stochastic input-output systems. Int. J. Syst. Sci. 13 (1982) $273-287$.

[41] P. Smith, Model misspecification in data envelopment analysis. Ann. Oper. Res. 73 (1997) 233-252.

[42] K. Tone, A slacks-based measure of efficiency in data envelopment analysis. Eur. J. Oper. Res. 130 (2001) $498-509$.

[43] P. Wanke, C.P. Barros and A. Emrouznejad, A comparison between stochastic DEA and fuzzy DEA approaches: revisiting efficiency in Angolan banks. RAIRO:OR 52 (2018) 285-303.

[44] M. Wen, C. You and R. Kang, A new ranking method to fuzzy data envelopment analysis. Comput. Math. App. 59 (2010) 3398-3404.

[45] M. Wen, Z. Qin and R. Kang, Sensitivity and stability analysis in fuzzy data envelopment analysis. Fuzzy Optim. Decis. Making 10 (2011) 1-10.

[46] M. Wen, Z. Qin, R. Kang and Y. Yang, Sensitivity and stability analysis of the additive model in uncertain data envelopment analysis. Soft Comput. 19 (2015) 1987-1996.

[47] L.A. Zadeh, Fuzzy sets. Inf. Control 8 (1965) 338-353.

[48] M. Zahedi-Seresht, G.R. Jahanshahloo and J. Jablonsky, A robust data envelopment analysis model with different scenarios. Appl. Math. Modell. 52 (2017) 306-319.

[49] Z. Zhou, E. Chen, H. Xiao, T. Ren and Q. Jin, Performance evaluation of portfolios with fuzzy returns. RAIRO:OR 53 (2019) 1581-1600. 